\title{
Weighted Local Times of a Sub-fractional Brownian Motion as Hida Distributions
}

\author{
Herry Pribawanto Suryawan \\ Department of Mathematics, Sanata Dharma University, Yogyakarta \\ E-mail: herrypribs@usd.ac.id
}

\begin{abstract}
The sub-fractional Brownian motion is a Gaussian extension of the Brownian motion. It has the properties of self-similarity, continuity of the sample paths, and short-range dependence, among others. The increments of sub-fractional Brownian motion is neither independent nor stationary. In this paper we study the sub-fractional Brownian motion using a white noise analysis approach. We recall the represention of sub-fractional Brownian motion on the white noise probability space and show that Donsker's delta functional of a sub-fractional Brownian motion is a Hida distribution. As a main result, we prove the existence of the weighted local times of a d-dimensional sub-fractional Brownian motion as Hida distributions.

Keywords: sub-fractional Brownian motion, local times, white noise analysis
\end{abstract}

\begin{abstract}
Abstrak
Gerak Brown subfraksional adalah proses Gaussian yang merupakan sebuah perluasan dari gerak Brown. Proses stokastik ini mempunyai beberapa sifat antara lain serupa diri, lintasan sampel yang kontinu, dan kebergantungan dalam jangkauan pendek. Kenaikan dari gerak Brown subfraksional tidak bersifat saling bebas ataupun stasioner. Di dalam makalah ini akan dipelajari gerak Brown subfraksional dengan menggunakan pendekatan analisis derau putih. Pertama, diingat kembali representasi gerak Brown subfraksional pada ruang peluang derau putih. Selanjutnya dibuktikan bahwa fungsional delta Donsker yang berkaitan merupakan distribusi Hida. Sebagai hasil utama, dibuktikan bahwa eksistensi dari waktu lokal terbobot dari sebuah gerak Brown subfraksional berdimensi $d$ adalah distribusi derau putih. Kata kunci: gerak Brown subfraksional, waktu lokal, analisis derau putih
\end{abstract}

\section{INTRODUCTION}

It is well-known that fractional Brownian (fBm) motion is an extension of the Brownian motion which satisfies Gaussian properties, self-similarity, long/short-range dependence, and stationarity of increments. Due to these properties $\mathrm{fBm}$ has been used as an important tool for stochastic modeling in hydrology, telecommunication, turbulence, image processing, finance, etc. A comprehensive study of $\mathrm{fBm}$ can be found in [1, 11] and references therein. Another extension of the Brownian motion is the so-called sub-fractional Brownian motion (sub-fBm). It was introduced by Bojdecki et al. [2] while studying occupation time fluctuations of branching

2000 Mathematics Subject Classification: 60H40, 60G15, 28C20, 46F 25 Received: 2019-08-26; Revised: 2020-01-19; accepted: 2020-02-02. 
particle systems with Poisson initial condition. The sub-fBm with parameter $H \in(0,1)$ is defined as a centered Gaussian process $S^{H}=\left(S^{H}(t)\right)_{t \geq 0}$ with $S^{H}(0)=0$ a.s. and covariance function

$$
\operatorname{Cov}\left(S^{H}(t), S^{H}(s)\right)=s^{2 H}+t^{2 H}-\frac{1}{2}\left((s+t)^{2 H}+|t-s|^{2 H}\right), \quad s, t \geq 0 .
$$

When $H=\frac{1}{2}$ one recovers the standard Brownian motion. $S^{H}$ is neither a semimartingale nor a Markov process unless $H=\frac{1}{2}$. This implies that the powerful techniques from classical stochastic calculus are not available when dealing with $S^{H}$. The sub-fBm shares many properties to those of $\mathrm{fBm}$ such as self-similarity and long-range dependence. On the contrary the increments of sub-fBm are not stationary. However, $S^{H}$ satisfies the inequalities, for any $s, t \geq 0$ with $t>s$,

$$
\left(\left(2-2^{2 H-1}\right) \wedge 1\right)(t-s)^{2 H} \leq \mathbb{E}\left(S^{H}(t)-S^{H}(s)\right)^{2} \leq\left(\left(2-2^{2 H-1}\right) \vee 1\right)(t-s)^{2 H} .
$$

Hence, by the Kolmogorov's continuity criterion, sample paths of sub-fBm is Hölder continuous of order $\gamma$ for any $\gamma<H$. As a conclusion sub-fBm is suitable for modeling of random phenomenon which posseses self-similarity, long/short dependence, continuous sample paths but non-stationary increments. Some works on sub-fBm can be found, for example, in $[3,4,5$, $10,13,14,15,16,17,18,19]$.

In this paper we deal with the problem of existence of the weighted local times of a sub$\mathrm{fBm}$ in higher dimensions. Our approach will be based on the theory of white noise analysis. The organization of the paper is as follow. In section 2 we summarize the basics of white noise theory including a realization of sub-fBm on the white noise probability space. Section 3 contains the main results of the paper on the existence of the weighted local times of a sub-fBm as Hida distributions.

\section{WHITE NOISE THEORY}

In this section we give some pertinent results of white noise analysis used throughout this paper. For a more comprehensive discussions including various applications of white noise theory we refer to $[7,9,12]$ and references therein. We start with the Gelfand triple

$$
\mathcal{S}_{d}(\mathbb{R}) \hookrightarrow L_{d}^{2}(\mathbb{R}) \hookrightarrow \mathcal{S}_{d}^{\prime}(\mathbb{R}),
$$

where $\mathcal{S}_{d}(\mathbb{R})$ is the space of $\mathbb{R}^{d}$-valued Schwartz test function, $\mathcal{S}_{d}^{\prime}(\mathbb{R})$ is the space of $\mathbb{R}^{d}$-valued tempered distributions, and $L_{d}^{2}(\mathbb{R})$ is the real Hilbert space of all $\mathbb{R}^{d}$-valued Lebesgue squareintegrable functions. Next, we construct a probability space $\left(\mathcal{S}_{d}^{\prime}(\mathbb{R}), \mathcal{C}, \mu\right)$ where $\mathcal{C}$ is the Borel $\sigma$-algebra generated by weak topology on $\mathcal{S}_{d}^{\prime}(\mathbb{R})$ and the probability measure $\mu$ is uniquely determined through the Bochner-Minlos theorem by fixing the characteristic function

$$
C(\vec{f}):=\int_{\mathcal{S}_{d}^{\prime}(\mathbb{R})} \exp (i\langle\vec{\omega}, \vec{f}\rangle) d \mu(\vec{\omega})=\exp \left(-\frac{1}{2}|\vec{f}|_{0}^{2}\right)
$$

for all $\vec{f} \in \mathcal{S}_{d}(\mathbb{R})$. Here $|\cdot|_{0}$ denotes the usual norm in the , and $\langle\cdot, \cdot\rangle$ denotes the dual pairing between $\mathcal{S}_{d}^{\prime}(\mathbb{R})$ and $\mathcal{S}_{d}(\mathbb{R})$. The dual pairing is considered as the bilinear extension of the inner product on $L_{d}^{2}(\mathbb{R})$, i.e.

$$
\langle\vec{g}, \vec{f}\rangle=\sum_{j=1}^{d} \int_{\mathbb{R}} g_{j}(x) f_{j}(x) d x,
$$

for all $\vec{g}=\left(g_{1}, \ldots, g_{d}\right) \in L_{d}^{2}(\mathbb{R})$ and $\vec{f}=\left(f_{1}, \ldots, f_{d}\right) \in \mathcal{S}_{d}(\mathbb{R})$. This probability space is known as the $\mathbb{R}^{d}$-valued white noise space since it contains the sample paths of the $d$-dimensional Gaussian white noise. In the white noise analysis setting a $d$-dimensional Brownian motion can be represented by a continuous modification of the stochastic process $B=\left(B_{t}\right)_{t \geq 0}$ with

$$
B(t):=\left(\left\langle\cdot, \mathbf{1}_{[0, t]}\right\rangle, \ldots,\left\langle\cdot, \mathbf{1}_{[0, t]}\right\rangle\right),
$$


such that for independent $d$-tuples of Gaussian white noise $\vec{\omega}=\left(\omega_{1}, \ldots, \omega_{d}\right) \in \mathcal{S}_{d}^{\prime}(\mathbb{R})$

$$
B(t, \vec{\omega})=\left(\left\langle\omega_{1}, \mathbf{1}_{[0, t]}\right\rangle, \ldots,\left\langle\omega_{d}, \mathbf{1}_{[0, t]}\right\rangle\right),
$$

where $\mathbf{1}_{A}$ denotes the indicator function of a set $A \subset \mathbb{R}$.

In order to represent sub-fBm on the white noise space, we use of the following operator

$$
M_{-}^{H} f:= \begin{cases}C_{H} D_{-}^{-\left(H-\frac{1}{2}\right)} f & \text { if } 0<H<\frac{1}{2} \\ f & \text { if } H=\frac{1}{2} \\ C_{H} I_{-}^{-\left(H-\frac{1}{2}\right)} f & \text { if } \frac{1}{2}<H<1,\end{cases}
$$

where $C_{H}=\sqrt{2 H \sin \pi H \Gamma(2 H)}$ dan $\Gamma$ denotes the gamma function. Here $I_{-}^{\beta} f, 0<\beta<1$ is the Weyl's type fractional integral operator defined by

$$
\left(I_{-}^{\beta} f\right)(x):=\frac{1}{\Gamma(\beta)} \int_{x}^{\infty} f(t)(t-s)^{\beta-1} d t
$$

and $D_{-}^{\beta} f, 0<\beta<1$ is the Marchaud's type fractional derivative operator defined by

$$
\left(D_{-}^{\beta} f\right)(x):=\lim _{h \rightarrow 0^{+}} \frac{\beta}{\Gamma(1-\beta)} \int_{h}^{\infty} \frac{f(x)-f(x+t)}{t^{1+\beta}} d t .
$$

For any Borel function $f$ on $[0, \infty)$ we define its odd extension $f^{\circ}$ by

$$
f^{\circ}(x)= \begin{cases}f(x) & \text { if } x \geq 0 \\ -f(-x) & \text { if } x<0\end{cases}
$$

Proposition 2.1. [17] It holds that $M_{-}^{H} \mathbf{1}_{[0, t]}^{\circ} \in L^{2}(\mathbb{R})$ and

$$
S^{H}(t)=\frac{1}{\sqrt{2}} \int_{\mathbb{R}}\left(M_{-}^{H} \mathbf{1}_{[0, t]}^{\circ}\right)(s) d B_{s},
$$

where $B=(B(t))_{t \in \mathbb{R}}$ is a one-dimensional two-sided Brownian motion.

As a consequence, the $d$-dimensional sub-fBm can be represented on the white noise space by a continuous modification of the stochastic process $S^{H}=(S(t))_{t \geq 0}$ with

$$
S^{H}(t):=\left(\left\langle\cdot, \frac{1}{\sqrt{2}} M_{-}^{H} \mathbf{1}_{[0, t]}^{\circ}\right\rangle, \ldots,\left\langle\cdot, \frac{1}{\sqrt{2}} M_{-}^{H} \mathbf{1}_{[0, t]}^{\circ}\right\rangle\right),
$$

such that for independent $d$-tuples of Gaussian white noise $\vec{\omega}=\left(\omega_{1}, \ldots, \omega_{d}\right) \in \mathcal{S}_{d}^{\prime}(\mathbb{R})$

$$
S^{H}(t, \vec{\omega})=\left(\left\langle\omega_{1}, \frac{1}{\sqrt{2}} M_{-}^{H} \mathbf{1}_{[0, t]}^{\circ}\right\rangle, \ldots,\left\langle\omega_{d}, \frac{1}{\sqrt{2}} M_{-}^{H} \mathbf{1}_{[0, t]}^{\circ}\right\rangle\right),
$$

where $\mathbf{1}_{A}^{\circ}$ denotes the odd extension of the indicator function of a set $A \subset \mathbb{R}$.

In the sequel we will use the Gel'fand triple

$$
(\mathcal{S}) \hookrightarrow L^{2}(\mu) \hookrightarrow(\mathcal{S})^{*}
$$

where $(\mathcal{S})$ is the space of white noise test functions obtained by taking the intersection of a family of Hilbert subspaces of $L^{2}(\mu)$. The space of white noise distributions $(\mathcal{S})^{*}$ is defined as the topological dual space of $(\mathcal{S})$. Elements of $(\mathcal{S})$ and $(\mathcal{S})^{*}$ are also known as Hida test functions and Hida distributions, respectively. The S-transform of an element $\Phi \in(\mathcal{S})^{*}$ is defined as

where

$$
(S \Phi)(\vec{f}):=\langle\langle\Phi,: \exp (\langle\cdot, \vec{f}\rangle):\rangle\rangle, \quad \vec{f} \in \mathcal{S}_{d}(\mathbb{R}),
$$

$$
: \exp (\langle\cdot, \vec{f}\rangle)::=\sum_{\mathbf{m} \in \mathbb{N}_{0}^{d}}\left\langle: \cdot \otimes^{\otimes \mathbf{m}}:, \vec{f}^{\otimes \mathbf{m}}\right\rangle=C(\vec{f}) \exp (\langle\cdot, \vec{f}\rangle),
$$

is the so-called Wick exponential and $\langle\langle\cdot, \cdot\rangle\rangle$ denotes the dual pairing between $(\mathcal{S})^{*}$ and $(\mathcal{S})$. We define this dual pairing as the bilinear extension of the sesquilinear inner product on $L^{2}(\mu)$. The S-transform provides a convenient way to identify a Hida distribution $\Phi \in(\mathcal{S})^{*}$, in particular, 
when it is hard to find the explicit form for the Wiener-Itô chaos decomposition of $\Phi$. Below is a sufficient condition on the Bochner integrability of a family of Hida distributions which depend on an additional parameter.

Theorem 2.2. [8] Let $(\Omega, \mathcal{A}, \nu)$ be a measure space and $\lambda \mapsto \Phi_{\lambda}$ be a mapping from $\Omega$ to $(\mathcal{S})^{*}$. If the S-transform of $\Phi_{\lambda}$ fulfils the following two conditions:

(1) the mapping $\lambda \mapsto S\left(\Phi_{\lambda}\right)(\vec{f})$ is measurable for all $\vec{f} \in \mathcal{S}_{d}(\mathbb{R})$, and

(2) there exist $C_{1}(\lambda) \in L^{1}(\Omega, \mathcal{A}, \nu), C_{2}(\lambda) \in L^{\infty}(\Omega, \mathcal{A}, \nu)$ and a continuous seminorm $\|\cdot\|$ on $\mathcal{S}_{d}(\mathbb{R})$ such that for all $z \in \mathbb{C}, \vec{f} \in \mathcal{S}_{d}(\mathbb{R})$

$$
\left|S\left(\Phi_{\lambda}\right)(z \vec{f})\right| \leq C_{1}(\lambda) \exp \left(C_{2}(\lambda)|z|^{2}\|\vec{f}\|^{2}\right)
$$

then $\Phi_{\lambda}$ is Bochner integrable with respect to some Hilbertian norm which topologizing $(\mathcal{S})^{*}$. Hence $\int_{\Omega} \Phi_{\lambda} d \nu(\lambda) \in(\mathcal{S})^{*}$, and furthermore

$$
S\left(\int_{\Omega} \Phi_{\lambda} d \nu(\lambda)\right)=\int_{\Omega} S\left(\Phi_{\lambda}\right) d \nu(\lambda) .
$$

\section{Main Results}

By using a limit argument and an Itô formula, Yan et al in [19] obtained a Tanaka formula for one-dimensional sub-fBm involving the so-called weighted local time of the form

$$
\mathcal{L}^{H}(x, T):=2 H\left(2-2^{2 H-1}\right) \int_{0}^{T} t^{2 H-1} \delta(X(t)-x) d t .
$$

Our aim is to define multidimensional version of $\mathcal{L}^{H}(x, T)$ in the framework of white noise analysis. First we show that the Donsker delta function of a sub-fBm exists in the sense of Hida distribution.

Theorem 3.1. Let $S^{H}=\left(S_{1}^{H}, \ldots, S_{d}^{H}\right)$ be a d-dimensional sub-fBm and $\vec{x} \in \mathbb{R}^{d}$. The Bochner integral

$$
\delta\left(S^{H}(t)-\vec{x}\right):=\frac{1}{(2 \pi)^{d}} \int_{\mathbb{R}^{d}} \exp \left(i\left\langle\vec{\lambda}, S^{H}(t)-\vec{x}\right\rangle\right) d \vec{\lambda}
$$

is a Hida distribution with the S-transform given by

$$
\begin{aligned}
& S \delta\left(S^{H}(t)-\vec{x}\right)(\vec{\varphi}) \\
& =\left(\frac{1}{2 \pi\left(2-2^{2 H-1}\right) t^{2 H}}\right)^{\frac{d}{2}} \exp \left(-\frac{1}{2\left(2-2^{2 H-1}\right) t^{2 H}} \sum_{j=1}^{d}\left(x_{j}-\left\langle\varphi_{j}, \frac{1}{\sqrt{2}} M_{-}^{H} \mathbf{1}_{[0, t]}^{\circ}\right\rangle\right)^{2}\right),
\end{aligned}
$$

for any $\vec{\varphi} \in \mathcal{S}_{d}(\mathbb{R})$.

Proof. For any $\vec{\varphi} \in \mathcal{S}_{d}(\mathbb{R})$ we have

$$
\begin{aligned}
& S \exp \left(i\left\langle\vec{\lambda}, S^{H}(t)-\vec{x}\right\rangle\right)(\vec{\varphi}) \\
& =\left\langle\left\langle\exp \left(i\left\langle\vec{\lambda}, S^{H}(t)-\vec{x}\right\rangle\right), C(\vec{\varphi}) \exp (\langle\cdot, \vec{\varphi}\rangle)\right\rangle\right\rangle \\
& =\exp \left(i(\vec{\lambda}, \vec{x})-\frac{1}{2}|\vec{\varphi}|_{0}^{2}\right) \int_{\mathcal{S}_{d}^{\prime}(\mathbb{R})} \exp \left(\left\langle\vec{\omega}, \vec{\varphi}-\frac{i \vec{\lambda}}{\sqrt{2}} M_{-}^{H} \mathbf{1}_{[0, t]}^{\circ}\right\rangle\right) d \mu(\vec{\omega}) \\
& =\exp \left(i(\vec{\lambda}, \vec{x})-\frac{1}{2}|\vec{\varphi}|_{0}^{2}\right) \exp \left(\frac{1}{2}\left|\vec{\varphi}-\frac{i \vec{\lambda}}{\sqrt{2}} M_{-}^{H} \mathbf{1}_{[0, t]}^{\circ}\right|_{0}^{2}\right) \\
& =\exp \left(i\left(\vec{\lambda}, \vec{x}-\left\langle\vec{\varphi}, \frac{1}{\sqrt{2}} M_{-}^{H} \mathbf{1}_{[0, t]}^{\circ}\right\rangle\right)-\frac{1}{2}|\vec{\lambda}|^{2}\left|\frac{1}{\sqrt{2}} M_{-}^{H} \mathbf{1}_{[0, t]}^{\circ}\right|_{0}^{2}\right)
\end{aligned}
$$


a measurable function of $\vec{\lambda} \in \mathbb{R}^{d}$. Furthermore, for $\vec{\varphi} \in \mathcal{S}_{d}(\mathbb{R})$ and $z \in \mathbb{C}$ we obtain

$$
\begin{aligned}
& \left|S \exp \left(i\left\langle\vec{\lambda}, S^{H}(t)-\vec{x}\right\rangle\right)(z \vec{\varphi})\right| \\
& \leq \prod_{j=1}^{d} \exp \left(-\frac{1}{2} \lambda_{j}^{2} \operatorname{Var}\left(S_{t}^{H}\right)\right) \exp \left(|z|\left|\lambda_{j}\right|\left|\left\langle\varphi_{j}, \frac{1}{\sqrt{2}} M_{-}^{H} \mathbf{1}_{[0, t]}^{\circ}\right\rangle\right|\right) \\
& \leq \prod_{j=1}^{d} \exp \left(-\frac{1}{2} \lambda_{j}^{2} \operatorname{Var}\left(S_{t}^{H}\right)\right) \exp \left(\frac{1}{4} \lambda_{j}^{2} \operatorname{Var}\left(S_{t}^{H}\right)\right) \exp \left(|z|^{2}\left|\varphi_{j}\right|^{2}\right) \\
& =\exp \left(-\frac{2-2^{2 H-1}}{4} t^{2 H}|\vec{\lambda}|^{2}\right) \exp \left(|z|^{2}|\vec{\varphi}|_{0}^{2}\right) .
\end{aligned}
$$

In the last expression the first factor is an integrable function of $\vec{\lambda}$ and the second factor is constant. Thus, according to Theorem 2.2 we have $\delta\left(S^{H}(t)-\vec{x}\right) \in(\mathcal{S})^{*}$. Finally, by applying Gaussian integral formula we obtain

$$
\begin{aligned}
& S \delta\left(S^{H}(t)-\vec{x}\right)(\vec{\varphi}) \\
& =\frac{1}{(2 \pi)^{d}} \int_{\mathbb{R}^{d}} S \exp \left(i\left\langle\vec{\lambda}, S^{H}(t)-\vec{x}\right\rangle\right)(\vec{\varphi}) d \vec{\lambda} \\
& =\prod_{j=1}^{d} \frac{1}{2 \pi} \int_{\mathbb{R}} \exp \left(i \lambda_{j}\left(x_{j}-\left\langle\varphi_{j}, \frac{1}{\sqrt{2}} M_{-}^{H} \mathbf{1}_{[0, t]}^{\circ}\right\rangle\right)-\frac{1}{2} \lambda_{j}^{2} \operatorname{Var}\left(S_{t}^{H}\right)\right) d \lambda_{j} \\
& =\prod_{j=1}^{d} \frac{1}{\sqrt{2 \pi \operatorname{Var}\left(S_{t}^{H}\right)}} \exp \left(-\frac{1}{2\left(2-2^{2 H-1}\right) t^{2 H}}\left(x_{j}-\left\langle\varphi_{j}, \frac{1}{\sqrt{2}} M_{-}^{H} \mathbf{1}_{[0, t]}^{\circ}\right\rangle\right)^{2}\right) \\
& =\left(\frac{1}{2 \pi\left(2-2^{2 H-1}\right) t^{2 H}}\right)^{\frac{d}{2}} \exp \left(-\frac{1}{2\left(2-2^{2 H-1}\right) t^{2 H}} \sum_{j=1}^{d}\left(x_{j}-\left\langle\varphi_{j}, \frac{1}{\sqrt{2}} M_{-}^{H} \mathbf{1}_{[0, t]}^{\circ}\right\rangle\right)^{2}\right) .
\end{aligned}
$$

Theorem 3.2. Let $S^{H}=\left(S_{1}^{H}, \ldots, S_{d}^{H}\right)$ be a d-dimensional sub-fBm and $\vec{x} \in \mathbb{R}^{d} \backslash\{\overrightarrow{0}\}$. Further, we assume that for any $\vec{\varphi}=\left(\varphi_{1}, \ldots, \varphi_{d}\right) \in \mathcal{S}_{d}(\mathbb{R})$ there exists $j \in\{1, \ldots, d\}$ fulfils

$$
\int_{\mathbb{R}} \varphi_{j}(x) M_{-}^{H} \mathbf{1}_{[0, t]}^{\circ}(x) d x \neq x_{j}
$$

Then, the Bochner integral

$$
\mathcal{L}^{H}(\vec{x}, T):=2 H\left(2-2^{2 H-1}\right) \int_{0}^{T} t^{2 H-1} \delta\left(S^{H}(t)-\vec{x}\right) d t .
$$

is a Hida distribution with the $S$-transform given by

$$
\begin{aligned}
& S \mathcal{L}^{H}(\vec{x}, T)(\vec{\varphi}) \\
& =\frac{2 H\left(2-2^{2 H-1}\right)^{1-\frac{d}{2}}}{(2 \pi)^{\frac{d}{2}}} \int_{0}^{T} t^{(2-d) H-1} \exp \left(-\frac{1}{2\left(2-2^{2 H-1}\right) t^{2 H}} \sum_{j=1}^{d}\left(x_{j}-\left\langle\varphi_{j}, \frac{1}{\sqrt{2}} M_{-}^{H} \mathbf{1}_{[0, t]}^{\circ}\right\rangle\right)^{2}\right) d t,
\end{aligned}
$$

for any $\vec{\varphi} \in \mathcal{S}_{d}(\mathbb{R})$.

Proof. Let us denote $\Psi_{t}:=t^{2 H-1} \delta\left(S^{H}(t)-\vec{x}\right)$. Then, for any $\vec{\varphi} \in \mathcal{S}_{d}(\mathbb{R})$ we have

$$
\begin{aligned}
& S \Psi_{t}(\vec{\varphi}) \\
& =\frac{t^{(2-d) H-1}}{\left(2 \pi\left(2-2^{2 H-1}\right)\right)^{\frac{d}{2}}} \exp \left(-\frac{1}{2\left(2-2^{2 H-1}\right) t^{2 H}} \sum_{j=1}^{d}\left(x_{j}-\left\langle\varphi_{j}, \frac{1}{\sqrt{2}} M_{-}^{H} \mathbf{1}_{[0, t]}^{\circ}\right\rangle\right)^{2}\right),
\end{aligned}
$$


is a measurable function of $t$. In the following, for simplicity, $2-2^{2 H-1}$ will be denoted by $\beta_{H}$. Next, for $\vec{\varphi} \in \mathcal{S}_{d}(\mathbb{R})$ and $z \in \mathbb{C}$ it holds

$$
\begin{aligned}
& \mid S \Psi_{t}(z \vec{\varphi} \mid \\
& \leq \frac{t^{(2-d) H-1}}{\left(2 \pi \beta_{H}\right)^{\frac{d}{2}}} \prod_{j=1}^{d} \exp \left(-\frac{1}{2 \beta_{H} t^{2 H}} x_{j}^{2}\right) \exp \left(\frac{\left|x_{j}\right||z|}{\beta_{H} t^{2 H}}\left|\left\langle\varphi_{j}, \frac{1}{\sqrt{2}} M_{-}^{H} \mathbf{1}_{[0, t]}^{\circ}\right\rangle\right|\right) \\
& \quad \times \exp \left(\frac{|z|^{2}}{2 \beta_{H} t^{2 H}}\left|\left\langle\varphi_{j}, \frac{1}{\sqrt{2}} M_{-}^{H} \mathbf{1}_{[0, t]}^{\circ}\right\rangle\right|^{2}\right) \\
& \leq \frac{t^{(2-d) H-1}}{\left(2 \pi \beta_{H}\right)^{\frac{d}{2}}} \exp \left(-\frac{|\vec{x}|^{2}}{4 \beta_{H} t^{2 H}}\right) \exp \left(\frac{3}{2}|z|^{2}|\vec{\varphi}|_{0}^{2}\right) .
\end{aligned}
$$

The factor $\exp \left(\frac{3}{2}|z|^{2}|\vec{\varphi}|_{0}^{2}\right)$ is a constant with respect to $t$, hence it is in $L^{\infty}([0, T], d t)$. On the other hand,

$$
\frac{t^{(2-d) H-1}}{\left(2 \pi \beta_{H}\right)^{\frac{d}{2}}} \exp \left(-\frac{|\vec{x}|^{2}}{4 \beta_{H} t^{2 H}}\right) \in L^{1}([0, T], d t) .
$$

To be more precise, by using formula $3.381(3)$ in [6] we obtain

$$
\int_{0}^{T} \frac{t^{(2-d) H-1}}{\left(2 \pi \beta_{H}\right)^{\frac{d}{2}}} \exp \left(-\frac{|\vec{x}|^{2}}{4 \beta_{H} t^{2 H}}\right) d t=\frac{1}{2 H} \frac{1}{\left(2 \pi \beta_{H}\right)^{\frac{d}{2}}}\left(\frac{4 \beta_{H}}{|\vec{x}|^{2}}\right)^{\frac{d-2}{2}} \Gamma\left(\frac{d-2}{2}, \frac{|\vec{x}|^{2}}{4 \beta_{H} T^{\frac{1}{2 H}}}\right),
$$

where $\Gamma(\cdot, \cdot)$ denotes the lower incomplete gamma function. An application of Theorem 2.2 finishes the proof of the theorem.

\section{CONCLUDING REMARKS}

The main result in this paper is an existence of the weighted local times of a $d$-dimensional sub-fBm at any spatial point $\vec{x} \neq \overrightarrow{0}$ in the sense of Hida distributions. As sub-fBm starts at $\overrightarrow{0}$ we expect a strong divergence of the weighted local times at point $\vec{x}=\overrightarrow{0}$. In fact, for the case $\vec{x}=\overrightarrow{0}$, the proof of Theorem 3.2 does not apply. One common way to solve this kind of problem is by a renormalization procedure. It is also of interest to drop or weaken the technical assumption (3) in Theorem 3.2. These will be considered for the forthcoming work.

\section{Acknowledgment}

The author would like to thank the Research and Service to Community Institute (LPPM), Universitas Sanata Dharma Yogyakarta, for the internal grant to fund this research. This grant aims to improve research and publication activities for researchers at Universitas Sanata Dharma.

\section{REFERENCES}

[1] Biagini, F., Hu, Y., Oksendal, B., and Zhang, T., 2008, Stochastic Calculus for Fractional Brownian Motion and Applications, Berlin: Springer-Verlag.

[2] Bojdecki, T., Gorostiza, L. G., and Talarczyk, A., 2004, Sub-fractional Brownian Motion and Its Relation to Occupation Times, Statist. Probab. lett., 69, 405-419.

[3] Bojdecki, T., Gorostiza, L. G., and Talarczyk, A., 2004, Fractional Brownian Density Process and Its Self-intersection Local Time of Order k, J. Theoret. Probab., 69, 717-739.

[4] Bojdecki, T., Gorostiza, L. G., and Talarczyk, A., 2007, Some Extensions of Fractional Brownian Motion and Sub-fractional Brownian Motion Related to Particle Systems, Elect. Comm. in Probab., 12, 161-172.

[5] El-Nouty, Ch., 2012, The Lower Classes of the Sub-fractional Brownian Motion, Stochastic Differential Equations and Processes, 7, 179-196. 
[6] Gradshteyn, I. S., and Ryzhik, I. M., 2015, Table of Integrals, Series, and Products, 8th ed., Boston: Academic Press.

[7] Hida, T. et al, 1993, White Noise. An Infinite Dimensional Calculus, Dordrecht: Kluwer Academic Publishers.

[8] Kondratiev, Y. et al, 1996, Generalized Functionals in Gaussian Spaces: The Characterization Theorem Revisited, J. Funct. Anal., 141, 301-318.

[9] Kuo, H-H., 1996, White Noise Distribution Theory, Boca Raton: CRC Press.

[10] Mendy, I., 2010, On the Local Time of Sub-fractional Brownian Motion, Annales Mathematiques Blaise Pascal, 17, 357-374.

[11] Mishura, Y., 2008, Stochastic Calculus for Fractional Brownian Motions and Related Processes, Berlin: Springer-Verlag.

[12] Obata, N., 1994, White Noise Calculus and Fock Space, Heidelberg: Springer Verlag.

[13] Tudor, C., 2007, Some Properties of the Sub-fractional Brownian Motion, Stochastics, 79, 431-448.

[14] Tudor, C., 2008, Inner Product Spaces of Integrands Associated to Subfractional Brownian Motion, Statist. Probab. Lett., 78, 2201-2209.

[15] Tudor, C., 2008, Some Aspects of Stochastic Calculus for the Sub-fractional Brownian Motion, Ann. Univ. Bucuresti, Mathematica, 2008, 199-230.

[16] Tudor, C., 2013, On the Wiener Integral with Respect to a Sub-fractional Brownian Motion on an Interval, J. Math. Anal. Appl., 351, 456-468.

[17] Wang, Z., and Yan, L., 2013, The S-Transform of Sub-fBm and an Application to a Class of Linear Subfractional BSDEs, Advances in Mathematical Physics, 2013, Article ID 827192, 11 pages.

[18] Yan, L., and Shen, G., 2010, On the Collision Local Time of Sub-fractional Brownian Motions, Statist. Probab. Lett., 80, 296-308.

[19] Yan, L., Shen, G., and He, K., 2011, Itô Formula for a Sub-fractional Brownian Motion, Comm. Stoch. Anal., 5, 135-159. 
